\title{
$\begin{array}{ll}\text { Research Square } & \text { Preprints are preliminary reports that have not undergone peer review. }\end{array}$ or referenced by the media as validated information.
}

\section{Multi-epitope peptide sequence in-silico construction from HGV genome}

\section{Kumar Sharp ( $\nabla$ ksharp0016@gmail.com )}

Government Medical College and Hospital, Jalgaon https://orcid.org/0000-0002-5034-8326

\section{Research Article}

Keywords: Hepatitis G, vaccine, hepatitis C, HIV, peptide, reverse vaccinology

Posted Date: August 6th, 2020

DOI: https://doi.org/10.21203/rs.3.rs-53866/v1

License: (c) (i) This work is licensed under a Creative Commons Attribution 4.0 International License. Read Full License 


\section{Abstract}

In this study I have approached through in-silico method or reverse vaccinology taking advantage of the genome sequence of hepatitis $\mathrm{G}$ virus. It serves its benefit of identifying antigens seen by both conventional as well as discovering any novel antigen. This peptide candidate can serve a triple purpose of hepatitis $C$ vaccine, hepatitis $G$ vaccine and HIV management addition. $89.2 \%$ of the residues were in the favoured region of Ramachandran plot. These points make it favourable for in-vitro trials and further refinement. Because of the high similarity of hepatitis $C$ genome to hepatitis $\mathrm{G}$ genome, it is highly probable that this peptide sequence might act as both hepatitis $C$ and hepatitis $G$ vaccine. Patients with past or current HGV infection have higher CD4+ lymphocyte counts and better AIDS-free survival rates. This peptide sequence might cause a breakthrough in the treatment of HIV without exposing them to develop hepatitis.

\section{Introduction}

In this study I have approached through in-silico method or reverse vaccinology taking advantage of the genome sequence of hepatitis $\mathrm{G}$ virus. It serves its benefit of identifying antigens seen by both conventional as well as discovering any novel antigen [1]. With technological advancement in the field of immunology these studies have become easier and more accurate [2]. This peptide candidate can serve a triple purpose of hepatitis $\mathrm{C}$ vaccine, hepatitis $\mathrm{G}$ vaccine and HIV management addition.

\section{Methodology}

The procedure used in this study is entirely based on two previous studies [3][4]. It will not be repeated here but is summarised below in figure 1 after obtaining necessary permission from its authors.

The modifications done in this study is as follows:

The genome sequence of hepatitis G virus was taken from NCBI virus database [5] (accession number:NC001710) Polyprotein precursor protein of this genome is used in this study. Docking of final peptide sequence was done with tertiary structure of toll-like receptor 7 obtained from Protein Data Bank [6] (PDB ID:5GMF).

\section{Result}

The final multi-epitope sequence formed after performing step 1 and 2 of methodology comprised of 214 amino acids:

GIINTLQKYYCRVRGGRCAVLSCLPKEEQIGKCSTRGRKCCRRKEAAAKAVEAGVT WYAAYLLDFVFVLLAAYVTDAVAAIQAAYDVALETELYGPGPGWPLYQAGLAVRP GKSGPGPGAASYLMGLGVGGNAQGPGPGPLYQAGLAVRPGKSAGPGPGAVFFSGL APLRMHPDGPGPGASYLMGLGVGGNAQTGPGPGVFFSGLAPLRMHPDV 
The above sequence distribution is as follows:

GIINTLQKYYCRVRGGRCAVLSCLPKEEQIGKCSTRGRKCCRRK EAAAK

(The above sequence is beta-defensin adjuvant sequence with EAAAK linker)

AVEAGVTWY AAY LLDFVFVLL AAY VTDAVAAIQ AAY DVALETELY GPGPG

(Polyprotein precursor CTL epitopes linked to each other by AAY linker with highest immunogenicity and at the end with GPGPG linker to HTL epitopes)

$\begin{array}{llll}\text { WPLYQAGLAVRPGKS } & \text { GPGPG } & \text { AASYLMGLGVGGNAQ } & \text { GPGPG } \\ \text { PLYQAGLAVRPGKSA } & \text { GPGPG } & \text { AVFFSGLAPLRMHPD } & \text { GPGPG }\end{array}$

ASYLMGLGVGGNAQT GPGPG VFFSGLAPLRMHPDV

(Polyprotein precursor HTL epitope with GPGPG linker)

The antigenicity prediction as in step 3 by Vaxijen server predicted it to be a probable antigen with score 0.462 . The allergenicity prediction as in step 3 by Algpred server predicted it to be a non-allergen with score of -0.86908598 (positive predictive value is $0 \%$ and negative predictive value is $0 \%$ ).

Physio-chemical properties as estimated by step 4 using ProtParam server gave the following results:

Molecular weight:21806.21 Daltons

Theoretical $\mathrm{pl}=9.28$

Estimated half-life in E. coli: >10 hours (in vivo)

Instability index: 27.48(stable)

Aliphatic index: 80.79

Grand average of hydropathicity (GRAVY): 0.096

The secondary structure of the final multi-epitope sequence was computed using PHYRE 2 server: $40 \%$ comprised of alpha-helix, $3 \%$ of beta-strand, $13 \%$ of transmembrane helix and $22 \%$ was disordered (Figure 2).

The tertiary structure obtained from PHYRE2 server was subjected to refinement by Galaxy Refine tool which generated 5 models as follows (Table 1): 


\begin{tabular}{|lllllll|}
\hline Model & GDT-HA & RMSD & MolProbity & Clash Score & Poor rotamers & Rama favoured \\
\hline Initial & 1.0000 & 0.000 & 4.192 & 141.9 & 10.2 & 73.1 \\
\hline Model 1 & 0.9276 & 0.473 & 2.239 & 12.1 & 0.7 & 85.8 \\
\hline Model 2 & 0.9241 & 0.487 & 2.281 & 11.8 & 1.4 & 88.2 \\
\hline Model 3 & 0.9241 & 0.483 & 2.092 & 8.9 & 0.0 & 87.3 \\
\hline Model 4 & 0.9287 & 0.461 & 2.167 & 12.1 & 0.0 & 89.2 \\
\hline Model 5 & 0.9077 & 0.499 & 2.231 & 13.1 & 0.7 & 87.7 \\
\hline
\end{tabular}

Table 1: Galaxy Refine structure models

Model 4 was chosen as the best tertiary structure of the sequence for further analysis. It was visualized using UCSF Chimera software [7] (Figure 3).

Ramachandran plot analysis by RAMPAGE server (Figure 4) gave the following result: Number of residues in favoured region ( 98.0\% expected): 189 (89.2\%); Number of residues in allowed region ( 2.0\% expected): 15 (7.1\%); Number of residues in outlier region: 8 (3.8\%)

The predicted B-cell linear epitopes were calculated using Ellipro suite (Figure 5 and 6).

Toll-like receptor 7 was docked with the final model by PatchDock server and top 10 results were refined using FireDock server. Solution number 2 was the most favourable binding conformation with global energy at -4.41 and 0.00 repulsive Vander Waal forces. The docked model was visualized using UCSF Chimera (Figure 7).

\section{Discussion}

The protein sequence is predicted to be antigenic as well as non-allergic, hence proving its advantage of not producing any harmful hypersensitivity reaction in the body. It is basic in nature and has low molecular weight hence suitable for any route of administration except oral. It's half-life in E. coli is $>10$ hours, hence can easily be cultured and extracted. It is thermally stable as indicated by instability index. It has various B-cell epitope stimulating site and molecular docking with toll like receptor TLR-7 shows that it binds easily it without any repulsive Van der Waal forces. Toll-like receptor 7 which induces immune response against ss-RNA organisms will elicit an immune response against this sequence considering it be an active virus and thus fulfilling its purpose as a vaccine. $89.2 \%$ of the residues were in the favoured region of Ramachandran plot. These points make it favourable for in-vitro trials and further refinement. All these studies were on web-tool prediction servers designed for such type of studies. Because of the high similarity of hepatitis $\mathrm{C}$ genome to hepatitis $\mathrm{G}$ genome, it is highly probable that this peptide sequence might act as both hepatitis $\mathrm{C}$ and hepatitis $\mathrm{G}$ vaccine [8]. Patients with past or current HGV 
infection have higher CD4+ lymphocyte counts and better AIDS-free survival rates [9][10][11]. This peptide sequence might cause a breakthrough in the treatment of HIV without exposing them to develop hepatitis. However, since they work on growing databases, these cannot give a complete surety for success in future stages. Along with the advantage of the study, there are some limitations. $22 \%$ of the predicted secondary structure is disordered. Instead of $98 \%$ proteins being in the favourable region of Ramachandran plot only $89.2 \%$ of them is present. Advanced molecular dynamic simulations were not performed like RMSD (root mean square deviation). These disadvantages need to be overcome with better resources but these early results do serve as a guiding path to build future work upon it.

\section{Conclusion}

This study has highlighted a potential candidate fulfilling its purpose as hepatitis $C$ vaccine, hepatitis $G$ vaccine and HIV management addition. In-depth studies and refinement might serve to be successful since it has very good results at such an early stage. It is needed to be validated experimentally.

\section{Declarations}

\section{Conflict of interest:}

The author declares no conflict of interest.

\section{Source of funding:}

Nil.

\section{Ethical consideration}

Not required.

\section{References}

1. Rappuoli R. Reverse vaccinology. Current opinion in microbiology. 2000 Oct 1;3(5):445-50.

2. Ali A, Khan A, Kaushik AC, Wang Y, Ali SS, Junaid M, Saleem S, Cho WC, Mao X, Wei DQ. Immunoinformatic and systems biology approaches to predict and validate peptide vaccines against Epstein-Barr virus (EBV). Scientific reports. 2019 Jan 24;9(1):1-2.

3. Khan S, Khan A, Rehman AU, Ahmad I, Ullah S, Khan AA, Ali SS, Afridi SG, Wei DQ. Immunoinformatics and structural vaccinology driven prediction of multi-epitope vaccine against Mayaro virus and validation through in-silico expression. Infection, Genetics and Evolution. 2019 Sep $1 ; 73: 390-400$. 
4. Sharp K, Dange S. Application of In-Silico Reverse Vaccinology for Designing Multi-Epitope Vaccine Against Coronavirus (preprint available on ChemRxiv) https://doi.org/10.26434/chemrxiv.12345653

5. Benson DA, Karsch-Mizrachi I, Lipman DJ, Ostell J, Sayers EW. GenBank. Nucleic acids research. 2011 Jan;39(Database issue):

6. Bank PD. Protein data bank. Nature New Biol. 1971; 233:223.

7. Pettersen EF, Goddard TD, Huang CC, Couch GS, Greenblatt DM, Meng EC, Ferrin TE. UCSF Chimeraa visualization system for exploratory research and analysis. Journal of computational chemistry. 2004 Oct;25(13):1605-12.

8. Kim JP, Fry KE. Molecular characterization of the hepatitis G virus. J Viral Hepat 1997; 4: 77-79

9. Toyoda H, Fukuda Y, Hayakawa T, Takamatsu J, Saito H. Effect of GB virus C/hepatitis G virus coinfection on the course of HIV infection in haemophilia patients in Japan. J Acquir Immune Defic Syndr Hum Retrovirol. 1998; 17:209-13.

10. Sabin CA, Devereux H, Kinson Z, Griffioen A, Brown D, Dusheiko G, et al. Effect of coinfection with hepatitis $\mathrm{G}$ virus on HIV disease progression in hemophilic men. J Acquir Immune Defic Syndr Hum Retrovirol. 1998;19: 546-8.

11. Lefre` re JJ, Roudot-Thoraval F, Morand-Joubert L, Petit JC, Lerable J, Thauvin M, et al. Carriage of $G B$ virus $C$ /hepatitis $G$ virus RNA is associated with a slower immunologic, virologic, and clinical progression of human immunodeficiency virus disease in coinfected persons. J Infect Dis. 1999;179: 783-9.

\section{Figures}




\section{Selection of Proteins}

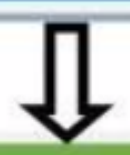

Prediction/selection of CTL and HTL epitopes

\section{II}

Calculating allergenicity and antigenicity of vaccine sequence

\section{II}

Reviewing physio-chemical properties of vaccine sequence

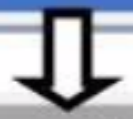

Prediction of secondary and tertiary structure of vaccine

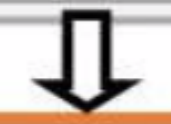

B-cell epitope prediction

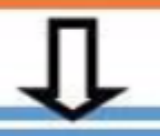

\section{Molecular docking with toll-like receptor}

\section{Figure 1}

Methodology summary from a similar previous study. Reproduced with permission from the authors. 


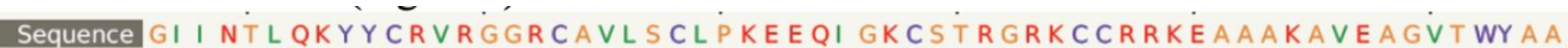
Secondary structure

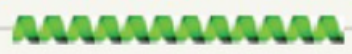

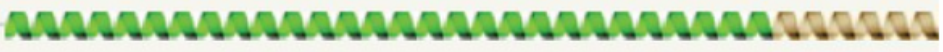

confidence

Disorder

Disorder

confidence

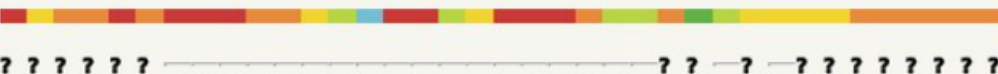

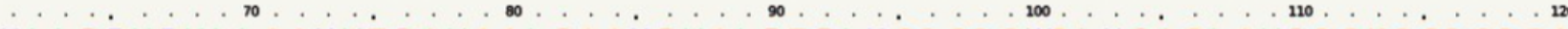
Sequence YLLDF VFVLLAAYVTDAVAAI QAA Y DVALETEL Y GPGPGWPL Y QAGLAVRPGKS GPGPGA Secondary structure

confidence

Disorder

Disorder

confidence

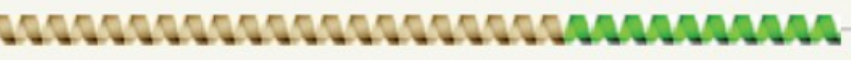

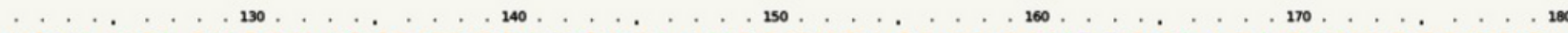

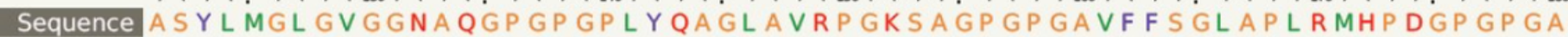
Secondary structure

confidence Disorder Disorder

confidence

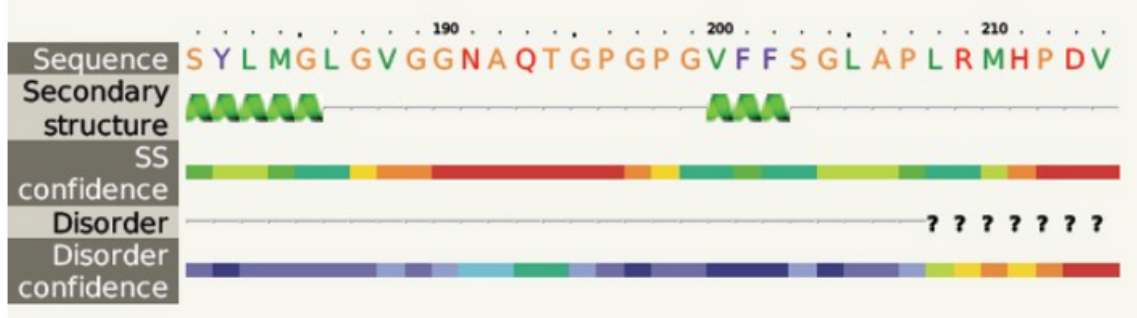

Confidence Key High(9) ] || Low (0)

? Disordered (22\%)

Malpha helix ( $40 \%$ )

$\Rightarrow$ Beta strand ( $3 \%)$

50. TM helix (13\%)

\section{Figure 2}

Secondary structure of vaccine sequence. 


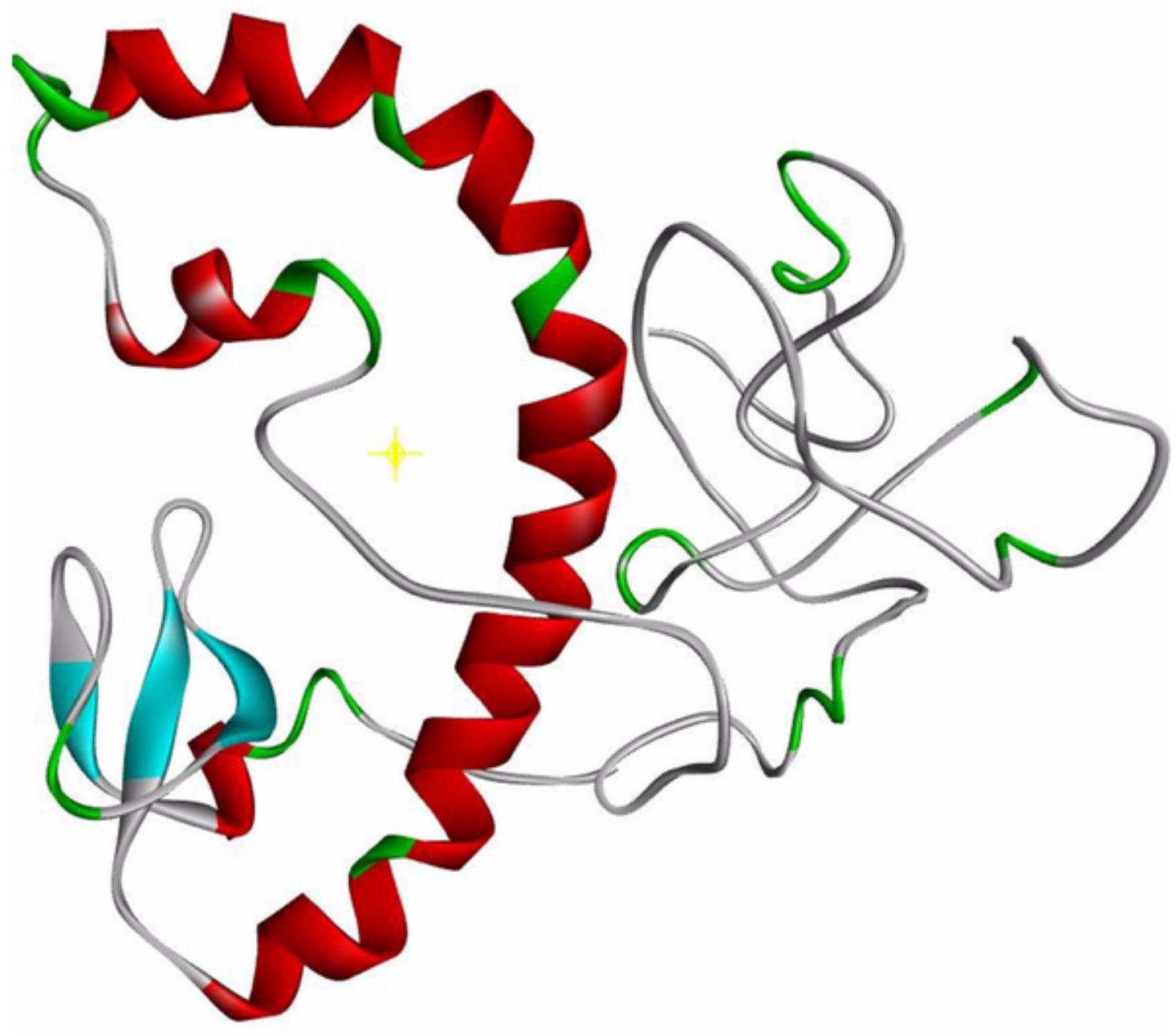

Figure 3

Model 4 tertiary structure.

Page 9/12 

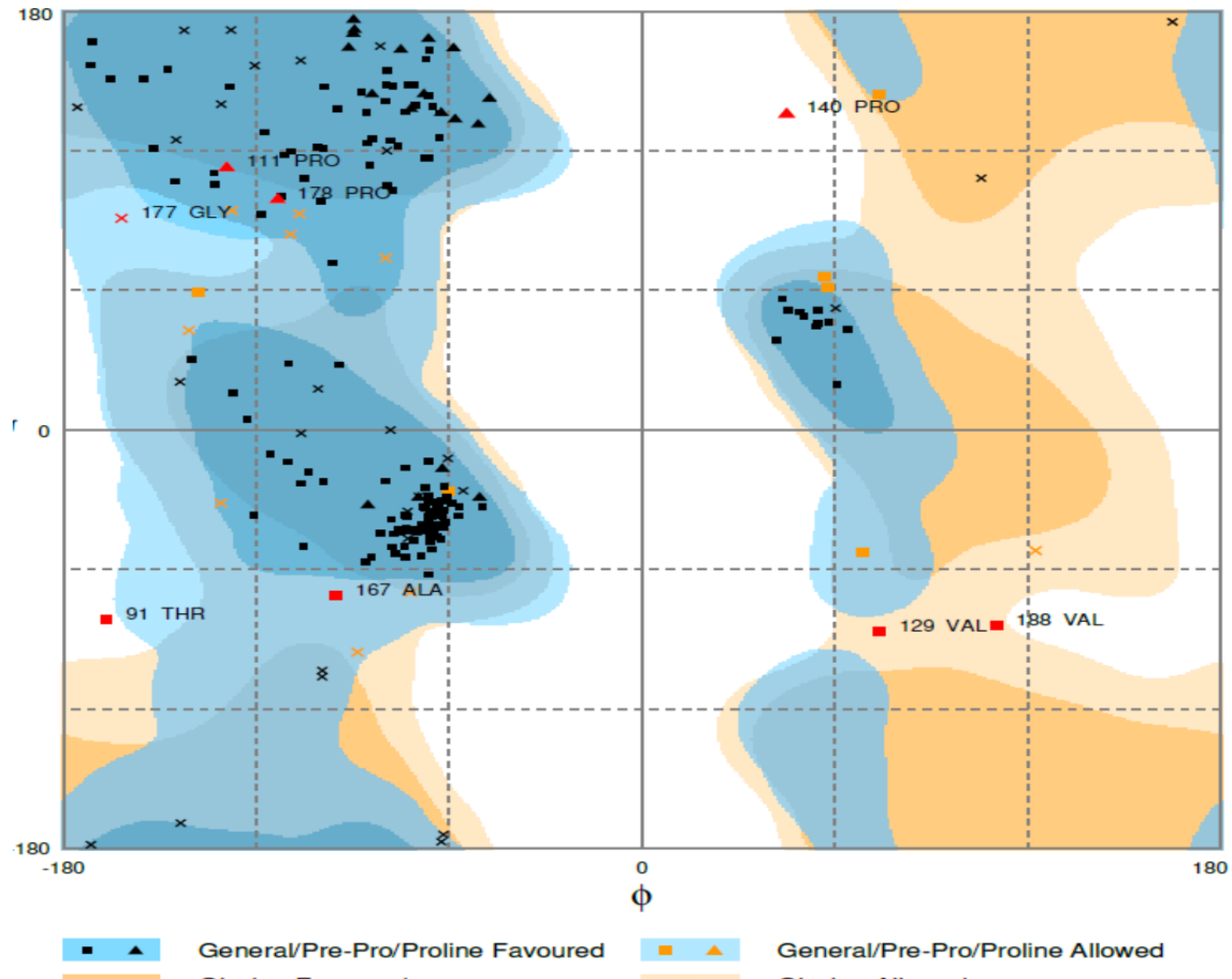
Glycine Favoured

General/Pre-Pro/Proline Allowed Glycine Allowed

Figure 4

Ramachandran Plot analysis of the final vaccine tertiary structure. 


\begin{tabular}{|c|c|c|c|c|c|c|}
\hline 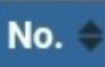 & Chain $\triangleq$ & Start $\curvearrowright$ & End $\triangleq$ & Peptide & Number of residues $\triangleq$ & Score \\
\hline 1 & - & 75 & 99 & TDAVAAIQAAYDVALETELYGPGPG & 25 & 0.815 \\
\hline 2 & - & 40 & 51 & CCRRKEAAAKAV & 12 & 0.782 \\
\hline 3 & - & 159 & 175 & GAVFFSGLAPLRMHPDG & 17 & 0.692 \\
\hline 4 & - & 1 & 28 & GIINTLQKYYCRVRGGRCAVLSCLPKEE & 28 & 0.662 \\
\hline 5 & - & 194 & 214 & TGPGPGVFFSGLAPLRMHPDV & 21 & 0.628 \\
\hline 6 & - & 138 & 145 & PGPLYQAG & 8 & 0.606 \\
\hline
\end{tabular}

\section{Figure 5}

B-cell linear epitopes predicted by Ellipro suite

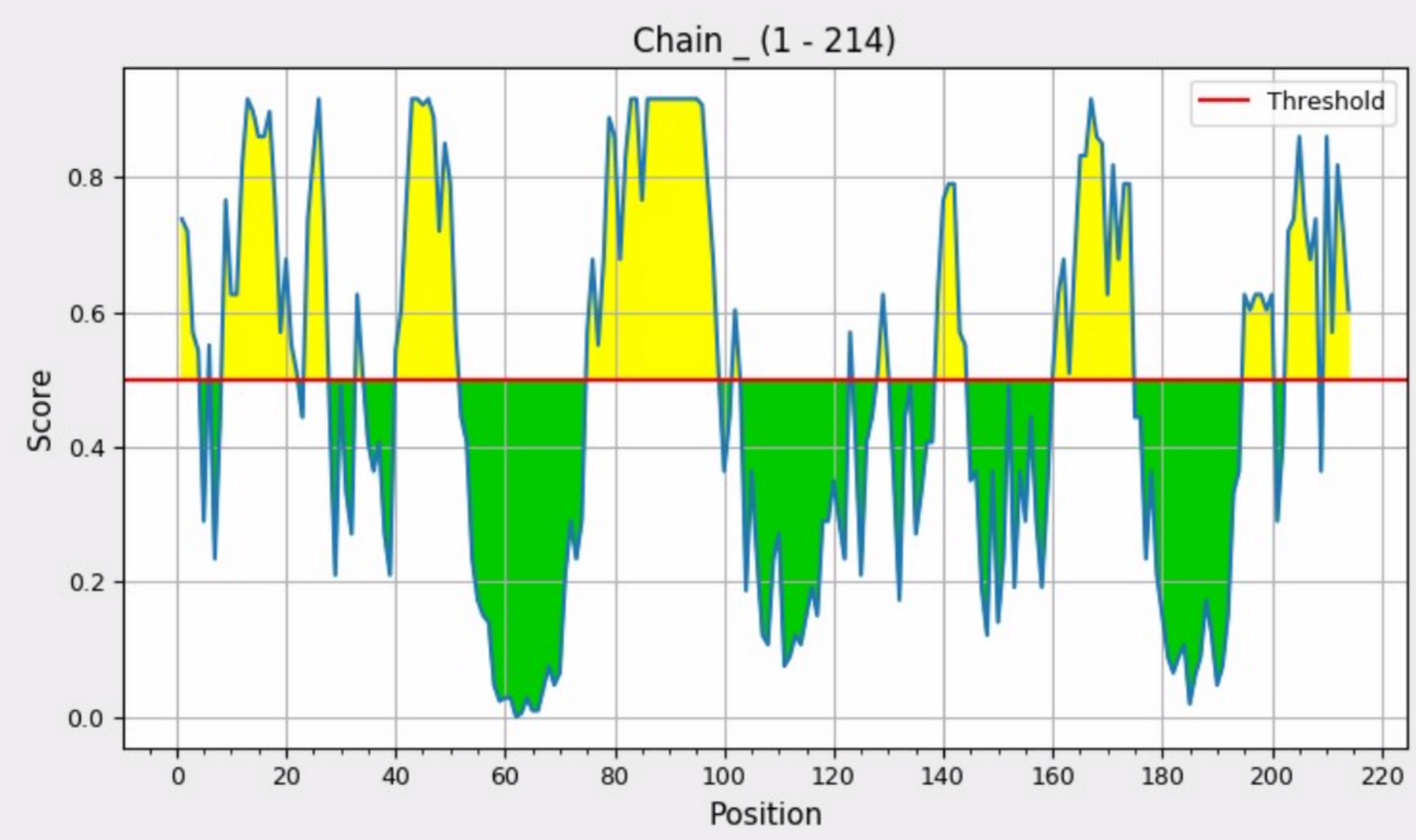

Figure 6

Epitope score chart from Ellipro suite 


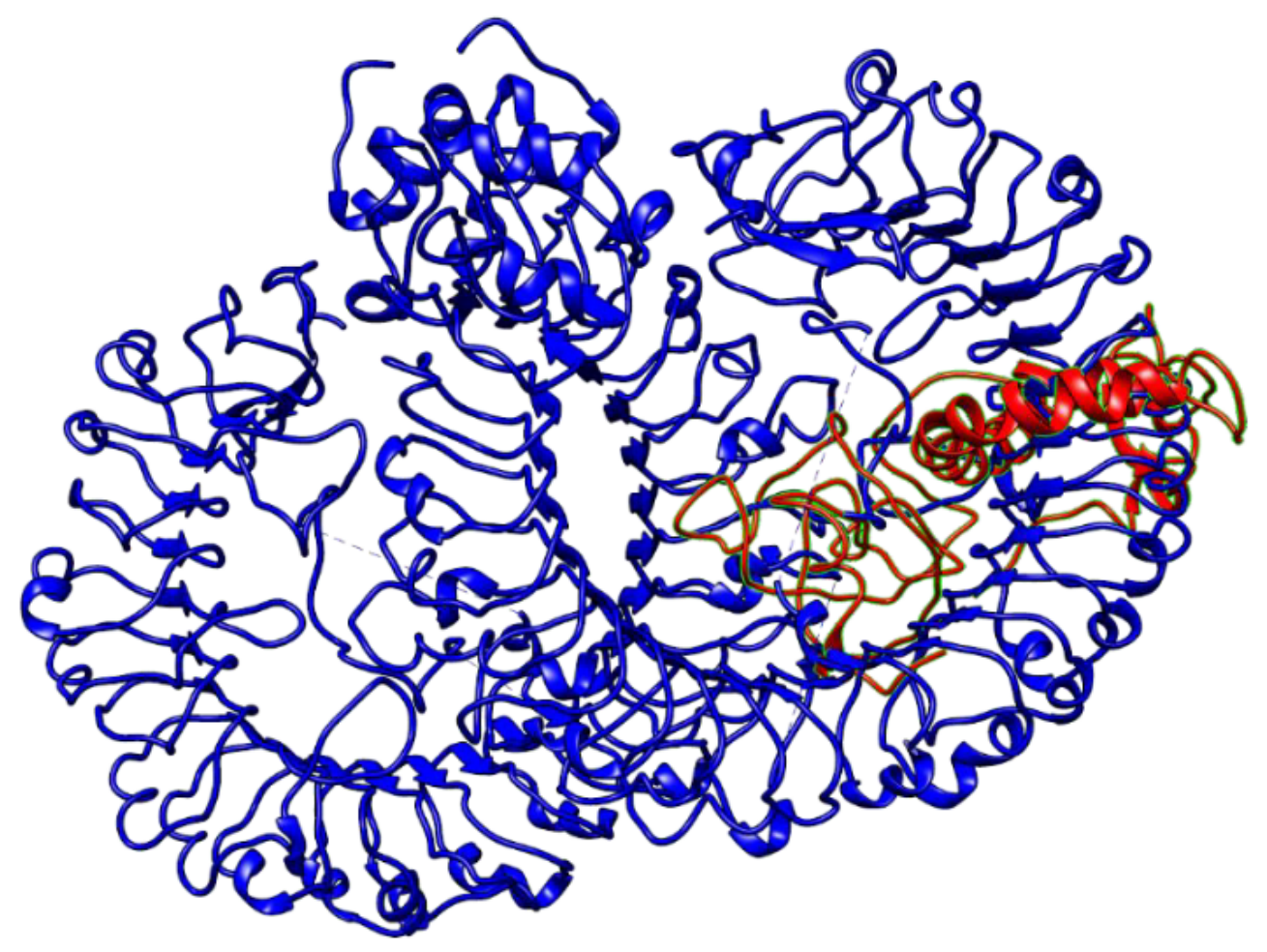

Figure 7

Peptide model(red) docked with Toll-like receptor 7(blue). 